\title{
Olfactory and Gustatory Function in Patients With Different Types of Maxillofacial Trauma
}

Kenza Drareni ${ }^{12}$, Thomas Hummel ${ }^{1}$, Moustafa Bensafi ${ }^{2}$, Charles-Arnaud Serex ${ }^{34}$, Marianne Hugentobler ${ }^{4}$, Joanne Rimmer ${ }^{56}$, Hergen Friedrich ${ }^{7}$, François Voruz ${ }^{34}$, Andrej Terzic ${ }^{3}$, Basile $\mathrm{N}$ Landis $^{4}$

- PMID: 32352171

- DOI: $10.1002 /$ lary.28701

\section{Abstract}

Objectives/hypothesis: To investigate olfactory and gustatory function in patients with maxillofacial trauma and associated fractures.

Study design: Retrospective cohort study.

Methods: Olfactory and gustatory function was assessed psychophysically in 124 patients who had sustained maxillofacial trauma with an associated fracture. Five groups were defined based on the fracture type: Le Fort, mandibular, nasal, orbital, and zygomatic. Olfaction was measured with Sniffin' Sticks (threshold, discrimination, identification [TDI] score) and gustation with the taste spray method. Patients selfrated olfactory and gustatory function on a visual analog scale prior to formal testing.

Results: Ten out of 124 patients were found to be anosmic (8\%), with half of them found in the Le Fort (skull base) group. The Le Fort fracture group had significantly lower olfactory function than other fracture types (TDI score $=22.4 \pm 10.7 ; \mathrm{P}=.01$; possible range $=1-48$ ). The mean gustatory spray test score was $3.82 \pm 0.4$ (possible range $=0-4$ ) without any intergroup differences. Self-rated olfactory function showed a correlation with the measured scores $(r=0.61, P<.001)$ across all groups.

Conclusions: The present data show a significant effect of maxillofacial fracture type on the development of anosmia. Maxillofacial fractures involving the skull base, such as Le Fort fractures, are more likely to cause permanent smell loss, whereas the other fracture types are rarely associated with anosmia. 